\title{
ORIGINAL
}

\section{SEROPREVALENCIA DE LA INFECCIÓN POR Borrelia Burgdorgferi Y Rickettsia Conorii EN POBLACIÓN HUMANA Y CANINA DE LA ZONA BÁSICA DE SALUD DE SAN ANDRÉS DEL RABANEDO (LEÓN, ESPAÑA)}

\author{
Jaime Rojo Vázquez \\ Servicios Veterinarios de Salud Pública. León
}

\section{RESUMEN}

Fundamentos: Se estudia la seroprevalencia de la infección por Borrelia burgdorferi y Rickettsia conorii en población humana y canina para conocer la situación de ambas en humanos, al mismo tiempo que la significación del perro, como indicador de la circulación de estos agentes entre aquéllos, en la Zona de Salud de San Andrés del Rabanedo, León.

Método: Se realizó un estudio en 98 sueros humanos y 95 caninos (de diversas razas y aptitudes) frente a $B$. burgdorferi (títulos de positividad $/ / 128$ y $1 / 64$, respectivamente) y 104 sueros humanos y 84 caninos frente a $R$. conorii (positividad a título 1/64 en ambas especies) mediante inmunofluorescencia indirecla (IFI).

Resultados: Se halló positividad a las dos infecciones, tanto en personas como en perros. Frente a $B$. burgdorferi fue superior en humanos que en perros y frente a $R$. conorii fue superior en éstos que en humanos. En personas fue del $4,08 \%$ frente a $B$. burgdorferi y $1 \%$ frente a $R$. conorii; en perros fue del $2.10 \%$ frente a $B$. burgdorferi y del $14.28 \%$ frente a $R$. conorii. Los valores más altos se hallaron en los meses de primavera-verano, salvo en el caso de $B$. burgdorferi en perros. $\mathrm{La}$ seroprevalencia fue mayor en perros dedicados al cuidado de ganado (ovino) que en los de caza y guarda.

Conclusiones: Los porcentajes de seroprevalencia hallados en nuestro trabajo, tanto en seres humanos como en caninos, considerados en el ámbilo territorial de una zona geográfica semi-rural de la provincia de León, han sido iguales o inferiores a los reseñados para otras provincias, incluida la totalidad de la de León. En perros se halló mayor seroprevalencia frente a $R$. comorii que frente a $B$. burgdorferi, lo que indica que es el agente más extendido en nuestra Provincia, cono han señalado otros autores. Los valores hallados en seres humanos frente a $B$. burgdorferi han sido más altos que en perros; la existencia de reacciones cruzadas con otros microorganismos ha podido influenciar estos resultados. Por ello, consideramos necesario realizar más estudios de prevalencia de estas infecciones para una vigilancia epidemiológica adecuada y control de estas zoonosis, dada su repercusión en salud pública.

Palabras clave: Rickettsia conorii. Borrelia burgdorferi. Inmunofluorescencia. Zoonosis. Seroprevalencia. Fiebre botonosa. Fiebre exantemática mediterránea. Borreliosis de Lyme.

\footnotetext{
Correspondencia:

Jaime Rojo Vázquez

Centro de Salud «Pinilla»

Burbia, 33

San Andrés del Rábanedo

24191 León.
}

\section{ABSTRACT \\ Seroprevalence of the Infection caused \\ by Borrelia Burgdorferi and Rickettsia Conorii in Human and Canine Population in the Basic Health Area of San Andrés del Rabanedo (León, Spain)}

Background: Positive results in infections of borrelia burgdorferi and Rickettsia conorii in human and canine population is studied in order to understand the situation of both in humans, and at the same time discover the importance of the dog as an indicator of these agents amongs those in the Health Area of San Andrés del Rabanedo, León.

Methods: A study was made of 98 human serums and 95 canine serums (dogs of different breeds and capabilities) as regards B. burgdorferi (positive results $1 / 128$ and $1 / 64$, respectively) and 104 human serums and 84 canine serums as regards $R$. conorii (postive results at range 1/64 in both species) by means of indirect Immunofluorescence (IFI).

Results: Positivity in both infections was discovered in both humans and dogs. With regard to $B$. burgdorferi it was higher in humans than in dogs and with regard to $R$. conorii it was higher in dogs than in humans. In humans it was $4.08 \%$ as regards $B$. burgdorferi and $1 \%$ with respect to $R$. conorii; in dogs it was $2,10 \%$ as regards $B$. burgdorferi and $14.28 \%$ regarding $R$. conorii. The highest values were discovered in the Spring-Summer months except in the case of $B$. burgdorferi in dogs. Serum prevalence was greater in dogs used to guard other animals (sheep) than those involved in hunting and security.

Conclusions: The percentages of posilive results discovered in our work, in humans as well as dogs, estimated in the territorial arca of a geographical zone in a semi-rural León province, were equal or inferior to those discovered in other provinces, including ours. In dogs there were larger positive results regar ding $R$. conorii than $B$. burgdorferi. which indicates that it is the most extended agent within our Province, as other authors have pointed out. The values discovered in humans as regards $B$. burgdorferi were higher than those in dogs: the existence of reactions crossed with other microorganisms may have influenced these results. For this reason. we consider it necessary to conduct more studies on the prevalence fo these infections to obtain an appropriate epidemiological surveillance and control of these zoonosis, given their impact on public health.

Key words: Rickettsia conorii. Borrelia burgdorferi. Inmunofluorescency. Zoonosis. Seroprevalence. Botonous fever. Mediterranean exantematic fever. Lyme's disease. 


\section{INTRODUCCIÓN}

Algunas enfermedades e infecciones humanas y animales como la Enfermedad de Lyme (EL) o Borreliosis de Lyme, causada por Borrelia burgdorferi, y la Fiebre exantemática mediterranea o fiebre botonosa (F.E.M.), causada por Ricketsia conorii, «nuevas zoonosis», problemas emergentes en Salud Pública, necesitan más estudios sobre su prevalencia para situarlas en el contexto de la epidemiología.

$\mathrm{Su}$ importancia viene dada por ser antropozoonosis (aunque el hombre no sea el hospedador principal de sus agentes etiológicos). En ellas el perro puede servir como indicador de la aparición de procesos en personas dada la estrecha relación de convivencia entre los dos y la posibilidad de que transporte el vector infectado a hábitats hu$\operatorname{manos}^{1-6}$.

La EL, conocida desde primeros de siglo, tiene distribución mundial, incluyendo Europa y España ${ }^{7,8}$; en España se tiene constancia de su presencia desde 1.977, aunque fue en 1.987 cuando se confirmó serológicamente el primer caso9. Posteriormente han sido abundantes los hallazgos serológicos, tanto de procesos clínicos como de la infección en humanos. En el hombre generalmente presenta un cuadro clínico multisistémico, y un espectro de manifestaciones clínicas muy amplio $^{10.11}$. Anda y cols. ${ }^{8}$, además de demostrar serológicamente la EL en la Península Ibérica, comprobaron que la forma neurológica es la más frecuente, seguida de la cutánea y cardíaca. Afecta tanto a personas que por su vinculación laboral están más expuestas a contagio (forestal) como a población general, de manera que su espectro socio-laboral es muy amplio.

B. burgdorferi se transmite al hombre por la picadura de la garrapata vector. En España, se ha comprobado que Ixodes ricinus es el vector fundamental ${ }^{2.9 .12-15}$. Su presentación es mayor en el norte y centro que en el sur de la Península, coincidiendo con la dis- tribución de $I$. ricinus, portador de la borrelia. No obstante, se ha descrito EL en otras zonas, por lo que puede que existan otros vectores implicados en la transmisión del agente $^{12.16}$.

En el perro, la EL es conocida desde $1.984^{3}$ y se asocia con un cuadro clínico de artritis (con cojeras y dificultad de movimientos), deformación de articulaciones, carditis, nefritis y fiebre ${ }^{17.18}$, aunque también se presentan otros síntomas ${ }^{19}$. En España fue diagnosticada por Font y cols. en $1.992^{20}$. Puede, además, transportar la garrapata vector al hombre, por lo que supone un riesgo epidemiológico importante ${ }^{6,21.22}$.

La FEM tiene distribución fundamentalmente en el área mediterránea, siendo más frecuente en los meses de verano y otoño. Se considera un proceso febril exantemático agudo, generalmente benigno en el hombre, caracterizado clínicamente por la presencia de lesión en el lugar donde mordió la garrapata («tache noire»), y que cursa con fiebre, cefalalgia, dolores musculares (siendo este síntoma bastante constante) y articulares (artromialgias difusas) $)^{23-25}$. En regiones endémicas existe evidencia de altas proporciones de sujetos seropositivos sin antecedentes clínicos de enfermedad ${ }^{25}$.

El agente, $R$. conorii, se transmite al hombre por la picadura de una garrapata vector (garrapata marrón del perro) $R$. sanguineus ${ }^{4,15,24}$.

En el perro la infección por $R$. conorii es clínicamente inaparente, siendo discutibles las escasas referencias clínicas ${ }^{6}$. En España se han señalado desde 1.982 casos en áreas del norte, costa mediterranea y región cen$\operatorname{tral}^{26}$.

El perro puede servir como indicador de la actividad de $R$. conorii en determinadas áreas de España ${ }^{4}$, en relación con la presencia del agente en la garrapata del perro $(R$. sanguineus). El incremento de la incidencia de la enfermedad en humanos se ha ligado con amplios contactos entre éstos y garrapatas infectadas procedentes del perro ${ }^{27}$. El pa- 
pel epidemiológico del perro es importante, ya que el contacto con ellos confirma un alto porcentaje de enfermedad en personas ${ }^{24.25,27}$, al transportar mecánicamente la garrapata al hombre. Por otra parte, puede servir ocasionalmente como reservorio de $R$. conorii debido a la ricketsemia posterior a la infección ${ }^{6}$.

Los estudios de prevalencia de la infección (en humanos y perros) van incrementándose en la Península, señal de la importancia que adquieren en Salud Públi$\mathrm{ca}^{2+4,6,20.28-40}$, incluida esta Comunidad Autónoma, tanto en personas ${ }^{25.27,41-44}$, como en animales ${ }^{30.45}$ o en ambos ${ }^{46}$, y en nuestra Provincia, realizados en perros ${ }^{26,47}$.

En consecuencia, nos propusimos conocer la prevalencia de estas dos infecciones en hombre y perro, en la Zona Básica de Salud de San Andrés del Rabanedo (León) a fin de comprobar la significación epidemiológica que alcanzan en la misma.

\section{MATERIAL Y MÉTODOS}

La Zona Básica de Salud de San Andrés del Rabanedo, semi-rural, está situada al noroeste de la Ciudad de León, y linda con clla. Tienc una superficie de $80 \mathrm{~km}^{2}$, comprende cuatro Municipios y 26 núcleos de población, con 28.499 habitantes $(7.972<17$ años; $16.330=18-63$ años; $4.197>65$ años), 14.635 son mujeres y 13.864 hombres, de los que el $14,3 \%$ está dedicados a la ganadería (de ellos el $22,63 \%$ tiene contacto con perros).

El estudio comprendió:

1. Muestreo de sueros humanos: se tomaron muestras de sangre a 98 personas para analizar la serología frente a $B$. burgdorferi (67 hombres y 31 mujeres), y 104 frente a $R$. conorii (59 hombres y 45 mujeres) Estas personas acudieron al Centro de Salud para una extracción requerida por su facultativo. De ellos, seis eran trabajadores del sector forestal. Una mujer manifestó haber sido picada por una garrapata unos días antes de la extracción.

A todos se les hizo anamnesis, incluyendo edad, sexo, trabajo y contacto con animales.

En dos ocasiones se extrajo sangre a más de un miembro de la familia.

2. Muestreo de sueros caninos: se comprobaron 95 sueros frente a $B$. burgdorferi (44 machos y 51 hembras), y 84 frente a $R$. conorii (39 machos y 45 hembras) correspondientes a 42 explotaciones, predominando 1-2 perros/explotación (10 y 18 explotaciones), disminuyendo hasta una en que había siete perros, y que pertenecían a diversas razas y aptitudes (pastor, fundamentalmente, caza y guarda).

Los tubos con la sangre permanecieron unas horas a temperatura de laboratorio desuerando y una noche en refrigeración. El suero obtenido fue congelado a $-20^{\circ} \mathrm{C}$ hasta el momento de la prueba. La toma de muestras se realizó entre abril de 1.993 y marzo de $1995^{48,51}$. Los sueros se analizaron por inmunofluorescencia indirecta con Borrelia burgdorferi B-31 y Rickettsia conorii como antígenos, usando un kit comercial (bioMerieux), siguiendo la metodología recomendada por el laboratorio. Se empleó un control positivo y negativo para cada antígeno.

Frente a $B$. burgdorferi se consideraron positivos los sueros humanos con título de $1 / 128^{36,38,49}$, y los caninos $>1 / 64^{1 /, 29,40,46,47,52}$. Frente a $R$. conorii de $1 / 40$ en personas y perros $4,6,26,27,30,33,39$.

\section{RESULTADOS}

Hemos hallado positividad frente a los dos antígenos estudiados, tanto en personas como en perros. Una persona fue positiva frente a los dos antígenos.

En personas, de los 98 sueros probados frente a $B$. burgdorferi cuatro, $4,08 \%$, fueron positivos (una mujer y tres hombres). Un 
hombre trabajaba en el sector forestal y a otro se le realizó analítica por padecer cansancio, febrícula nocturna y mialgias. $\mathrm{La}$ sangre se extrajo en el período primaveraverano. Por otra parte, en el $0,96 \%$ de aquéllas (1 suero de 104) se hallaron anticuerpos frente a $R$. conorii: un varón de 40 años, que fue positivo también frente a $B$. burgdorferi. La sangre se extrajo en primavera. La mujer mordida por una garrapata, mostró un título de anticucrpos por debajo del umbral considerado (Tabla 1).

En perros, el 2,10\% tenía anticuerpos frente a $B$. burgdorferi (2 de 95). Uno cuidaba ganado ovino y convivía con otros de los que extrajo sangre por ser peligrosos; el otro guardaba ganado bovino y convivía con otros que fueron negativos. Las muestras de sangre se extrajeron en invierno. Frente a $R$. conorii el $14,28 \%$ de los perros (12 de 84) fue positivo: $11(13,09 \%)$ eran pastores ( 8 de ovino, 3 de vacuno) y 1 $(1,19 \%)$ de caza. En una explotación el pero que dio resultado positivo estaba solo; en dos, los dos perros de la misma fucron positivos, y en siete había varios animales de los que uno sólo resultó positivo. Ningún perro reaccionó frente a dos antígenos. La sangre fue extraída en verano y otoño (Tabla 2 ).

No hubo correspondencia en la positividad persona/perro en los casos en que ambos convivían.

\section{DISCUSIÓN}

Frente a $B$. burgdorferi, la seroprevalencia hallada fue mayor en humanos que en perros, cuando debiera ser superior en éstos, o por lo menos igual, tratándose de una antropozoonosis $^{46}$, lo que confirmaría la relación entre la infección humana y la canina en la zona, si bien indica el mayor contacto de éstos con el agente, no necesariamente la infección ${ }^{3}$. La existencia de reacciones cruzadas con otras espiroquetas y microorganismos, patógenos o no ${ }^{7.50 .53}$, puede haber influenciado estos resultados, de tal manera que deberán ser analizados con cautela. Otra explicación podría estar en la existencia de una población escasa de garrapatas vectores, así como una baja población de garrapatas infectadas.

Tabla 1

Resultados obtenidos en los sueros humanos

\begin{tabular}{|lcccccc|}
\hline & Positivos & $\%$ & Negativos & $\%$ & Total & $\%$ \\
\hline $\begin{array}{l}\text { B. burgdorferi } \\
(98 \text { sueros: } 1 / 128)\end{array}$ & $\begin{array}{c}4 \\
(1 \mathrm{H}: 3 \mathrm{M})\end{array}$ & 4,08 & 94 & 95,92 & 98 & 100 \\
\hline $\begin{array}{l}\text { R. conorii } \\
(104 \text { sueros: } 1 / 40)\end{array}$ & $\begin{array}{c}1 \\
(\mathrm{M})\end{array}$ & 0,96 & 103 & 99,08 & 104 & 100 \\
\hline
\end{tabular}

Tabla 2

Resultados obtenidos en los sueros caninos

\begin{tabular}{|lcccccc|}
\hline & Positivos & $\%$ & Negativos & $\%$ & Total & $\%$ \\
\hline $\begin{array}{l}\text { B. } \text { burgdorferi } \\
(95 \text { sueros: } 1 / 64)\end{array}$ & $\begin{array}{c}2 \\
\text { (pastor) }\end{array}$ & 2,10 & 93 & 97,90 & 95 & 100 \\
\hline $\begin{array}{l}\text { R. conorii } \\
(84 \text { sueros: } 1 / 40)\end{array}$ & $\begin{array}{c}12 \\
(11 \mathrm{P} ; 1 \mathrm{C})\end{array}$ & 14,28 & 72 & 85,72 & 84 & 100 \\
\hline
\end{tabular}


Entre los perros, hemos hallado mayor seroprevalencia frente a $B$. burgdorferi en los pastores que en los de caza y guarda, al igual que otros autores ${ }^{4}$. Los pcrros analizados mostraron mayor reactividad serológica que las personas frente a $R$. conorii $(14,28 \%$ y $0,96 \%$, respectivamente). La seroprevalencia hallada en seres humanos en nuestra zona, muy inferior a la hallada en la Comunidad Autónoma (Soria, 5\%) 27.54 y sobre todo a la de zonas mediterráneas ${ }^{24,39,48.55 .56}$. hace pensar que existe bajo número de garrapatas parasitadas por $R$. conorii circulando entre perro y hombre, poco acceso del hombre a hábitats caninos o al revés, existiendo poco contacto perro-hombre, ya que el nivel de los anticuerpos en personas no parecen descender de forma rápida ${ }^{39}$.

La positividad serológica frente a $R$. conorii en los perros se halló en los meses de verano y primavera. La estacionalidad de los anticuerpos frente a este agente tras la picadura de la garrapata, ha sido señalada también por otros autores ${ }^{6.30 .39 .47}$.

Los valores de seroprevalencia encontrados en perros frente a $R$. conorii $(14,28 \%)$ son inferiores a otros señalados en la Península, aunque referidos a zonas endémicas (Mediterráneo y zona central) (.6. $30.33 \cdot 39,40$. En nuestra provincia Delgado y Cármenes ${ }^{47}$ haIlan una seroprevalencia del $22,0 \%$. Ia interferencia con otras especies de rickettsias $^{+7}$ y el muestreo en zonas del sur de la misma, de clima más benigno, pueden explicar las diferencias. En otros países, los valores son próximos ${ }^{57.58}$, aunque también dobles ${ }^{59}$.

Al igual que estos autores ${ }^{26,47}$, la seroprevalencia en perros frente a $B$. burgdorferi y $R$. conorii hallada por nosotros, pone de manifiesto la mayor difusión del segundo de estos agentes en esta provincia, si bien las diferencias en los valores que obtienen son menos acusadas.

Comparativamente, la positividad frente a $B$. burgdorferi en trabajadores del sector forestal analizados no fue superior a la de la población general, como cabría esperar ${ }^{38}$.

Los valores de seroprevalencia hallados frente a $B$. burgdorferi en población española, están comprendidos entre el $0.3 \%$ y $14,8 \%^{28,32,34,36,44,46,60}$ con valores próximos al nuestro $(4,08 \%)$ en La Rioja, Sevilla y Guadalajara. En la provincia de León $^{41}$ se ha hallado un porcentaje de personas reaccionantes que es el doble del que obtuvimos nosotros, aun con título de positividad cuatro veces superior al nuestro. En población de riesgo, lógicamente, los valores son muy superiores $^{31,37,38,42,60,61}$.

En otros países la seroprevalencia hallada, con valores extremos amplios $(1,8 \%$ $19,68 \%$ ), es mayoritariamente superior a la nuestra ${ }^{62-73}$.

Los resultados hallados frente a $B$. burgdorferi en perros $(2,10 \%)$, relativamente bajos, pueden tener la misma explicación que los hallados en personas: escasa población de garrapatas vectores, población de garrapatas infectadas baja, poco acceso de perros a focos naturales de infección y consiguiente exposición al agente, incluso por la poca difusión del agente en la zona, así como la posibilidad de reacciones cruzadas. Si bien próxima a la hallada en Madrid (1\%) ${ }^{35}$, es inferior a la hallada tanto en esta Comunidad Autónoma (16,43\% en Soria) y nuestra provincia $(20 \%)^{45-47} \mathrm{y}$ Madrid (perros infestados con garrapatas: $11,5 \%)^{40}$, como en otras zonas, europeas o mundiales $\mathrm{s}^{3,19,68,72,74-80}$.

El haber hallado individuos seropositivos en los meses de invierno frente a $B$. burgdorferi, induce a pensar que los anticuerpos frente a este antígeno persisten tras la picadura de la garrapata infectada, al contrario que sucede con $R$. conori $i^{39}$.

De los resultados de nuestro estudio se considera que son necesarias más investigaciones sobre estas infecciones para una confirmación más exacta y lograr una adecuada vigilancia epidemiológica y control de estas zoonosis, por su repercusión en salud pública. 


\section{BIBLIOGRAFÍA}

1. Buxton and Frazier. Animal Microbiology. Nueva York: Academic Press, 1977.

2. Campbe11, RS F. Pathogenesis and pathology of the complex rickettsial infections. Vet Bull 1.994;64(1):1-24.

3. Falco, R.C. y cols., 1.993, The distribution of canine exposure to Borrelia burgdorferi in a Lyme disease endemic area. Amer J Public Health 83(9): 1305-1310.

4. Herrero, C. y cols., 1.992. Evidence of the presence of spotted fever group rickettsiae in dogs and dogs-ticks of the central provinces in Spain. Eur J Epidemiol 8(4): 575-579.

5. Kelly, P.J., Mason, P.R., 1.991. Tick-bite fever in Zimbabwe. Survey of antibodies to Rickettsia conorii in man and dogs, and rickettsia-like organisms in dogs ticks. S Afri Med J 80:233-236.

6. Suárez Ferreiro, P. y cols., 1.992. Encuesta seroepidemiológica de la infección por Rickettsia conorii(F.E.M.) en el perro. Med Vet 9(9): 521-526.

7. Guerrero Espejo, A., La enfermedad de Lyme en España. Med Clin(Barc.) 1992, 98: 96-97.

8. Anda, P., y cols., A serological survey and review of clinical Lyme borreliosis in Spain. Clin Inf Dis 1993;16:310-319.

9. Oteo Revuelta, J. A. y Estrada Peña, A., Ixodes ricinus, vector comprobado de Borrelia burgdorferi en España. Med Clin(Barc.) 1.990, 95 (15), nov 3.

10. Guerrero, A., y cols., Borreliosis de Lyme. ¿Cómo se manifiesta en España? Med Clin (Barc.) 1993;101:5-7.

11. Guerrero, A., Manifestaciones clínicas de la enfermedad de Lyme. III Simp. SIGE'I, Alcalá de Henares, Madrid, 1.995.

12. Barral, M. y cols., 1.995. Aislamiento e identificación de Borrerelia burgdorferi en Ixodes ricinus del país vasco. III Symp. sobre ixodoidea y enf. transmitidas. Alcalá de Henares, Madrid.

13. Estrada Peña, A., Oteo Revuelta, J.A., 1.991. Los ixodidos vectores de Borrelia burgdorferi en el Nordeste español, con notas sobre la Borreliosis de Lyme en España. SIGET.

14. Quereda, C., 1.995. La enfermedad de Lyme en España. III Symposium SIGET, Alcalá de Henares, noviembre.
15. Sáez, A., Sánchez Covisa, A., Guerrero Espeso, A., 1.991. Garrapatas y enfermedad de Lyme. Enf. inf. y microbiol. clin., 9(2): 67-71.

16. Oteo Revuelta, J.A. Epidemiología y epizootiología de la infección por Borrelia burgdorferi. Primer Symp. SIGET, Zaragoza, diciembre.

17. Levy, J.A., and Dreesen, D.W., 1.992. Lyme borreliosis in dogs. Canine practice 17(2):

18. Stevens, A., Lyme borreliosis in domestic animals. Connecticut medicine, june, 1989, 53(6): 352-353.

19. Azuma, Y., 1.994. Canine Lyme disease: clinical and serological evaluations in 21 dogs in Japan. Vet Rec 134(15): 369-372.

20. Font, A., y cols., 1.992. Lyme disease in dogs in Spain. Vet. rec., 130: 227-228.

21. Lindenmayer, J.M. y cols, 1.991. Dogs as sentinels for Lyme disease in Massachsetts. Amer J Public Health 81(11): 1.448-1455.

22. Mather, TN., 1.994. Competence of dogs as reservoir for Lyme disease spirochetes (Borrelia burgdorferi). JAVMA 205, 2: 186-188.

23. Acha, P.N. y Szifres, B., 1.986. Zoonosis y enfermedades transmisibles comunes al hombre y a los animales. O.P.S., pub. científica No. 503, 2. ${ }^{\text {e ed. }}$ Washington.

24. Roca Arbones, V., 1.992. Fiebre botonosa mediterränea. Aspectos clínicos y terapéuticos. Bol Inf Com 2(1): 137-140.

25. Herrero Herrero, J.J., y Ruiz Beltrán, J., La fiebre exantemática mediterranea. Junta de Castilla y León. 1994.

26. Delgado, S. y Cármenes, P., 1.995. Canine seroprevalence of Rickettsia conorii infection (Mediterranean Spotted fever) in Castilla y León ( $\mathrm{Nw}$ Spain). Eur J Epidemiol 11: 1-4.

27. Saz, J.V., y cols., 1.993. Seroprevalence of Coxiella burnetti and $\mathrm{R}$. conorii infection in the province of Soria. Enf Inf Microbiol Clin 11(9): 469-473

28. Benito Ruesca, R., y cols., 1.991. Detección de anticuerpos anti-Borrelia burgdorferi mediante inmunofluorescencia indirecta. ler. Symposio SIGET, Zaragoza.

29. Caride, E. y cols., 1.995. Prevalencia de la borreliosis canina en Madrid. III Symp. ibér. Ixodoidea y enf. transmitidas. Alcalá de Henares, Madrid.

30. Espejo, E. y cols., 1.993, Antibodies to Rickettsia conorii in dogs: seasonal differences. Eur J Epidemiol 9(3): 344-346. 
31. Ferrero Cáncer, M., Benito Ruesca, R., Rubio Calvo, M.C., 1.991. Anticuerpos anti-Botrelia burgdorferi en donantes de Huesca. SIGET, com. $\mathrm{n} .{ }^{\circ} 8$.

32. Jiménez, A., y cols., 1.995. Seroprevalencia de la enfermedad de Lyme en la provincia de Guadalajara: comparación del estudio de dos muestras poblacionales. III Symp. ibér. Ixodoidea y enf. transmitidas.

33. Herrero Martín, C., 1.989. Estudio de Rickettsia conorii en perros de diversas localidades del Centro de España. Cong. Nac. Microbiología.

34. López Prieto, M.D., Borobio, M.V., 1.989. Prevalencia de anticuerpos frente a Borrelia burgdorferi en la población de Sevilla. Enf Inf Microbiol Clin 7(9): 489-490

35. Olmeda García, A.S., y cols., 1.991. Presencia de la infección por Borrelia burgdorferi (Enfermedad de Lyme) en perros en la provincia de Madrid. SIGET.

36. Oteo RevueltA. I.A., y cols., 1.990. Fnfermedad de Lyme en la Rioja. Med Clin (Barc.) 95 (15): noviembre.

37. Otco Revuclta, J.A. y cols., 1.992. Epidemiology and prevalence of seropositivity against B.burgdorferi antigen in La Rioja, Spain. Rev Epidemiol Santé Publique 40(2): 85-92.

38. Oteo Revuelta, J.A. y cols., 1.992. Prevalencia de anticuerpos frente a Borrelia Burgdorferi en una población de riesgo. Simp. COFAL sobre enf. de Lyme. Madrid. enero.

39. Segura, F. y cols., 1.995. Nuevos aspectos epidemiológicos de la infección por $\mathrm{R}$. conorii. III Symp. ibér. Ixodoidea y enf. transmitidas. Alcalá de Henares, Madrid, noviembre.

40. Tesouro, M.A., y cols., 1.995. Valoración inmunológica frente a Erlichia canis, Borrelia burgdorferi y Rickettsia conorii en perros de la Comunidad Autónoma de Madrid infestados por garrapatas. III Symp. ibér. Ixodoidea y enf. transmitidas. Alcalá de Henares, Madrid, noviembre.

41. Gegúndez, M., y cols., 1.995. Seroprevalencia de la enfermedad de Lyme en el Norte de Castilla y León. III Symp. ibér. Ixodoidea y enf. transmitidas.

42. Rodríguez Torres, A., y cols., 1.991. Borreliosis de Lyme. An R Acad Med Cir Valladolid XXIX, enero-abril: 65-70.

43. Saz, J.V., y cols., 1.994. Lyme disease in the province of Soria: clínico-epidemiologic study. Enf Inf Microbiol Clin 12(2): 52-59.
44. Tamayo, J. C., y cols., Anticuerpos frente a Borrelia burgdorferi en un grupo de población de Valladolid. Enf Inf Microbiol Clin 1990; 8: 663-664.

45. Serrano, J.L., y cols., 1.994. Estudio epidemiológico y seroprevalencia de anticuerpos frente a Borrelia burgdorferi en una muestra de población canina de la provincia de Soria. I Cong. Vet. Castilla y León, León.

46. Saz, J.V., y cols., 1.995. Enfermedad de Lyme: relación entre población humana y canina en la provincia de Soria. III Symp. ibér. Ixodoidea y enf. transmitidas. Alcalá de Henares, Madrid, novicmbre.

47. Delgado, S. y Cármenes, P., 1.995. Seroepidemiological survey for Borrelia burgdorferi (Lyme disease) in dogs from northwestern of Spain. Eur $\mathbf{J}$ Epidemiol 11:321-324.

48. Babalis, T. y cols., 1.993. Rickettsia conorii in Greece: comparison of a microinmunofluorescece assay and western blotting for seroepidemiology. Am J Trop Med Hyg 48(6):784-792.

49. Braden, Ch.R. y Klempner, M.S., s/a, Pruebas serológicas para el diagnóstico de la enfermedad de Lyme. Inf Dis Clin Practice, ed. español, 1(1): $10-17$.

50. Escudero, R. y cols., 1.993, La serología en la infección por Borrelia burgdorferi. Bol Contr cal 5(4): 108-116.

51. Greene, R.T. y cols., 1.991. Comparison of a enzyme-linked inmunosorbent assay o an indirect inmunofluorescence assay for the detection of antibodies to Borrelia burgdorferi in the dog. Vet Microbiol 26(1-2):179-190.

52. Magnarelli, L.A. y cols., Borreliosis in dogs from Southern Connekticut. JAVMA 1.985;186:955-959.

53. Shin, S.J., y cols., 1993. Cross-reactivity betwen B. burgdorferi and other spirochetes affects specifity of serotets for detection of antibodies to the Lyme disease agent in dogs. Vet Microbiol 36:161-174.

54. Boletín epidemiológico semanal, Min. de Sanidad y Consumo, Madrid, 1.992. Núm. 1.929.

55. Gourgouci, K. y cols., 1.992. Epidemiologycal research on Mediterranean spotted fever. Acta Microbiol Hellenica, 37(2):241-246.

56. Radulovic, S. y cols., 1.993. Prevalence of antibodies to spotted fever group rickettsiae along the aester coast of the Adriatic sea. J Clin Microbiol $31(8): 2225-2227$. 
57. Breitschwerdt, E.B. y cols., 1.987. Antibodies to spotted fever-group rickettssiae in dogs in North Carolina. Am J Vet Res 48(10): 1436.

58. Magnarelli, L.A. y cols., Antibodies to spotted fever-group rickettsiae in dogs and prevalence of infected ticks in southern Connecticut. Am $\mathbf{J}$ Vet Res 43(4): 656 .

59. Mandola, M.L., Massero, L., Gerbi, C., 1.992. Prevalence of antibodies to Rickettsia conorii in the canine population of the Asti area, Italy, following cases of M.S.F.. Nuovo progreso veterinario, $47(17)$ : $534-536$.

60. Oteo Revuelta, J.A., 1.994. Borreliosis de Lyme. Junta de Castilla y León. Imp. Heraldo de Zamora. Zamora.

61. Elías, C., y cols., 1.991. Estudio de las pruebas serológicas de la enfermedad de Lyme en un grupo de pacientes infectados por el virus de la inmunodeficiencia humana. 1er. Symposio SIGET, 7aragnza.

62. Anaud; CM. y cols., 1.993. Lyme disease: seroprevalence and vector survey in Southern Alberta. Canada Communicable disease Report, 19(24): 205-209.

63. Cristofolini, A., y cols., 1.993, Zoonoses transmited by ticks in forest workers (tick-borne encephalitis and Lyme borreliosis): preliminary results. Med Lav 84(5):394-402.

64. Fahrer, H. y cols., 1.991, The prevalence and incidence of clinical and asymptomatic Lyme borreliosis in a population at risk. J Inf Dis 163(2): 305-310.

65. Hassler, D. y cols., 1.992. Lyme borreliosis in a endemic region in Europe. Prevalence of antibodies and clinical spectrum. Dtsch. med. Wochenschr, 117(20): 767-774.

66. Kuiper, H. y cols., 1.991. Lyme borreliosis in dutch forestry workers. J Inf 23(3): 279-286.

67. Nohlmans, MKE. y cols., 1.991. Prevalence of Lyme borreliosis in the Netherlands. Netherlands Tijdschrift voor Gemeskunde, 135 (48): 2258-2292.

68. Pan, L.N., 1.991. The discover of Lyme disease in Fujian Province. Chung-Hua-Liu-Hsing-PingHsueh-Tsa-Chih., 12(1): 14.
69. Satanchi, N.O., Balaguel.J., 1.993. Lyme disease antibodies against Borrelia burgdorferi in farm workers in Argentina. Rev Saude Publica 27(4): 305-307.

70. Smith, H.V., Gray, J.S., Mckenzie, G., 1.991. A Lyme borreliosis human serosurvey of asyntomatic adults in Ireland. Int J Med Microbiol 275(3): 382-389.

71. Smidt, R., 1.986. Erythema Chronicum migrans disease in the Federal Republic of Germany. $\mathrm{Zbl}$ Bakt Hyg A 263, 435-441.

72. Treml, F., y cols., 1.994. Borrelia infection. Serological diagnosis of Lyme disease. Veterinarstvi. 44(6): $262-263$

73. Zivanovic, B., Ler, Z., Cekanac, R., Risk of infection with Borrelia burgdorferi in an area endemic from Lyme disease. Vojnosanit-Pregl., 48 (5): $400-404$.

74. Arashima, Y., 1.991. Anti-Borrelia burgdorferi antibody in dogs: lyme disease as zoonosis. Riusho Byori, 39(8): 869-874.

75. Artsob, H., y cols., 1.993. Serological studies on the infection of dogs in Ontario with Borrelia burgdorferi, the aetiological agent of Lyme disease. Canadian Vet J 34(9): 543-548

76. Costerg-Meot, F., 1.989. La maladie de Lyme. Enquête serologique chez le chien. Comparison de deux techniques: inmunofluorescence indirecte et westem blot. These doctorale. E.N.V.'Lyon.

77. Greene, R.T. y cols., 1.988. Antibodies to Borrelia burgdorferi in dogs in North Carolina. Am J Vet Res 49(4):473-476.

78. Hansen, K., Dietz, H.H., 1.989. Serosurvey for antibodies to Borrelia burgdorferi in Danish dogs. Acta Pathol Microbiol Inmunol Scand 97(3): 281 285.

79. Skardona, I. y cols., 1.993. Lyme disease in dogs: update on clinical diagnosis. Veterinarstvi $43(4)$ : 127

80. Weber, A. y cols., 1.991. Incidence of antibodies to Borrelia burgdorferi in dogs in small animal practice in North Bavaria. Berl Munch Tierarzt Woch 104(11): 384-386. 